\title{
Eficácia anti-helmíntica e desempenho em rebanho de matrizes ovinas na mesorregião leste do Rio Grande do Norte
}

\author{
[Anthelmintic efficacy and performance in herds of ewes in the eastern mesoregion of Rio Grande \\ do Norte]
}

\section{"Artigo Científico/Scientific Article"}

\author{
Francisca Fernanda Silva Roberto ${ }^{1}$, Gelson Santos Difante ${ }^{2}$, Antonio Leandro Chaves Gurgel ${ }^{2 *}$, \\ Lilian Gioto Zaros ${ }^{3}$, Henrique Rocha Medeiros ${ }^{4}$, Luiz Silva Vieira ${ }^{5}$
}

\author{
${ }^{1}$ Programa de Pós-Graduação em Zootecnia, Universidade Federal da Paraíba, Areia-PB, Brasil. \\ ${ }^{2}$ Programa de Pós-Graduação em Ciência Animal, Universidade Federal de Mato Grosso de Sul, Campo Grande-MS, Brasil. \\ ${ }^{3}$ Departamento de Microbiologia e Parasitologia, Universidade Federal do Rio Grande do Norte, Natal-RN, Brasil. \\ ${ }^{4}$ Unidade Acadêmica Especializada em Ciências Agrárias, Universidade Federal do Rio Grande do Norte, Macaíba-RN, Brasil. \\ ${ }^{5}$ Embrapa Caprinos e Ovinos, Sobral - CE, Brasil. \\ *Autor para correspondência/Corresponding author: E-mail: antonioleandro09@ gmail.com
}

\begin{abstract}
Resumo
Objetivou-se avaliar a eficácia anti-helmíntica em rebanhos de matrizes ovinas na mesorregião leste do Rio Grande do Norte. Os animais foram distribuídos em cinco grupos com 12 ovelhas cada, sendo estes: cloridrato de levamisol $(5 \mathrm{mg} / \mathrm{kg})$, closantel $(10 \mathrm{mg} / \mathrm{kg})$, moxidectina $(0,2 \mathrm{mg} / \mathrm{kg})$ e ivermectina $(0,2 \mathrm{mg} / \mathrm{kg})$ e o grupo controle sem tratamento anti-helmíntico. Foram avaliadas as seguintes variáveis no dia zero e a cada sete dias após a administração dos anti-helmínticos: contagens de ovos por grama de fezes (OPG), calculado o percentual de eficácia, peso, ganho de peso, escore de condição corporal, e o grau de anemia. Observou-se diferença significativa na OPG dos animais, onde as maiores médias foram observadas no grupo tratado com ivermectina $(7858,3 \pm 6880)$. A ivermectina $(34,7 \%)$ apresentou-se com baixa eficácia anti-helmíntica em relação a moxidectina $(93,3 \%)$, closantel $(89,1 \%)$ e ao levamisol $(89,4 \%)$. O peso final dos animais não foi alterado pelo uso dos anti-helmínticos, com média de $39,5 \mathrm{~kg}$. As matrizes tratadas com ivermectina, levamisol e o grupo controle, apresentaram-se com o escore de condição corporal concentrados nos graus 2,5 e 3 . Os graus de anemia ficaram dispostos entre 2 e 4 , sendo que o grupo medicado com a ivermectina foi o que apresentou animais com uma maior dispersão nesse intervalo. A moxidectina mostrou-se eficiente na redução de nematoides gastrintestinais no rebanho estudado e pode ser indicado no controle desses parasitos.
\end{abstract}

Palavras-chave: Helmintos gastrintestinais; ovelhas; resistência parasitária; teste de eficácia.

\begin{abstract}
The objective was to evaluate the anthelmintic efficacy in herds of ewes in the eastern mesoregion of Rio Grande do Norte. The animals were distributed in five groups with 12 sheep each, being: levamisole hydrochloride $(5 \mathrm{mg} / \mathrm{kg})$, closantel $(10 \mathrm{mg} / \mathrm{kg})$, moxidectin $(0.2 \mathrm{mg} / \mathrm{kg})$, ivermectin $(0.2 \mathrm{mg} / \mathrm{kg})$, and control group without anti-helminthic treatment. The following variables were evaluated at day zero and every seven days after administration of anthelmintics: egg counts per gram of feces (OPG), percentage of efficacy, weight, weight gain, body condition score, and the degree of anemia. A significant difference was observed in the OPG of the animals, where the highest averages were observed in the group treated with ivermectin $(7858.3 \pm 6880)$. Ivermectin (34.7\%) presented low anthelmintic efficacy in relation to moxidectin (93.3\%), closantel (89.1\%) and levamisole (89.4\%). The final weight of the animals was not altered by the use of anthelmintics, with a mean of $39.5 \mathrm{~kg}$. The ewes treated with ivermectin, levamisole, and the control group had a body condition score concentrated in grades 2.5 and 3. The degrees of anemia were distributed between 2 and 4 , and the group medicated with ivermectin had animals with a greater dispersion in this interval. Moxidectin was efficient in the reduction of gastrointestinal nematodes in the studied herd and may be indicated in the control of these parasites.
\end{abstract}

Keywords: Gastrointestinal helminths; sheeps; parasitic resistance; efficacy test. 


\section{Introdução}

A região Nordeste possui o maior rebanho de ovinos do Brasil, com aproximadamente 18.410.551 cabeças, sendo que o estado do Rio Grande do Norte encontra-se na quinta posição quando comparado a outros estados do Nordeste, com efetivo total de 864.048 cabeças (IBGE, 2017). A atividade está passando por uma fase de consolidação na região e representa uma importante fonte de renda para pequenos produtores oriundos de agricultura familiar no estado Rio Grande do Norte, sendo que na maioria das propriedades esses animais são criados em sistema extensivo.

Dentre os principais problemas na exploração de pequenos ruminantes, está a presença de nematoides gastrintestinais, uma vez que se aderem à mucosa do trato gastrintestinal, provocando lesões, dificultando o processo de absorção dos nutrientes e comprometendo o desempenho zootécnico dos mesmos. Os principais parasitos de ovinos são do gênero Haemonchus que podem representar até $80 \%$ de prevalência, seguido por Trichostrongylus e Strongyloides, e em menor porcentagem pelo Oesophagostomum (Ahid et al., 2008; Costa et al., 2011a). A influência da helmintose gastrintestinal no desempenho dos animais é nítida, pois provoca diminuição na produção, atraso no desenvolvimento e elevada mortalidade do rebanho (Pinheiro et al., 2000), além de alterações nas taxas reprodutivas das matrizes.

Para minimizar esses efeitos no rebanho, os produtores utilizam produtos químicos, geralmente de forma indiscriminada, sem se atentarem aos efeitos adversos do uso inadequado dessa estratégia (Amarante, 2009). Quando esses produtos são utilizados com frequência e doses superiores àquelas recomendadas pelos fabricantes podem acelerar o processo de resistência dos parasitos (Molento, 2004). Essas práticas podem resultar na seleção de populações resistentes aos diversos grupos químicos utilizados no tratamento dos animais (Melo et al., 2015), sendo necessário realizar testes de eficácia desses antiparasitários, cujos resultados auxiliarão na tomada de decisão de manejo sanitário do rebanho.

Nesse contexto, objetivou-se avaliar a eficácia anti-helmíntica de produtos químicos utilizados rotineiramente no controle de parasitos gastrintestinais em matrizes ovinas, e o efeito da eficácia no desempenho das matrizes na fase de pós aleitamento.

\section{Material e Métodos}

$\mathrm{O}$ experimento foi realizado na área do Grupo de Estudos em Forragicultura (GEFOR), situado na Unidade Acadêmica Especializada em Ciências Agrárias, pertencente à Universidade Federal do Rio Grande do Norte - UFRN em Macaíba, RN, na mesorregião Leste Potiguar ( $5^{\circ} .53$ ' 35.12" sul e $35^{\circ} 21^{\prime} 47.03$ " oeste). O clima da região é caracterizado como sub-úmido seco (Thornthwaite, 1948), durante o período experimental, no mês de março de 2017, a precipitação acumulada foi de $150 \mathrm{~mm}$ e a temperatura média de $28^{\circ} \mathrm{C}$ (INMET, 2017).

Foram utilizadas 60 matrizes ovinas, distribuídas aleatoriamente em cinco grupos com 12 matrizes cada (Tabela 1), naturalmente infectadas, sem padrão racial definido (SPRD), no pós aleitamento, com idade entre dois e quatro anos, peso médio inicial de $36,3 \mathrm{~kg} \pm 4,04$, há 60 dias sem tratamento anti-helmíntico e com contagem de OPG acima de 500 ovos. Os animais foram mantidos em pastos de capim-marandu, manejado sob lotação contínua durante o dia e abrigados em galpão em baia coletiva durante a noite, onde recebiam suplemento concentrado proteico/energético fixado em $0,5 \%$ do seu peso vivo, calculado para exigência da categoria (NRC, 2007).

Tabela 1. Disposição dos tratamentos avaliados.

\begin{tabular}{cccc}
\hline Grupo & Produto químico & Dosagem & Via de administração \\
\hline Grupo 1 (G1) & cloridrato de levamisol $5 \%$ & $5 \mathrm{mg} / \mathrm{kg}$ & via oral \\
Grupo 2 $(\mathrm{G} 2)$ & closantel $10 \%$ & $10 \mathrm{mg} / \mathrm{kg}$ & via oral \\
Grupo 3 (G3) & moxidectina $1 \%$ & $0,2 \mathrm{mg} / \mathrm{kg}$ & via subcutânea \\
Grupo 4 (G4) & ivermectina 0,08\% & $0,2 \mathrm{mg} / \mathrm{kg}$ & via oral \\
Grupo 5 (G5) & sem tratamento anti-helmíntico & - & - \\
\hline
\end{tabular}

As amostras de fezes foram coletadas diretamente da ampola retal dos animais e colocadas em sacos plásticos, ésteres, devidamente identificadas e acondicionadas em caixas 
isotérmicas para transporte ao laboratório para o processamento. As coletas foram feitas nos dias zero (D0), sete (D7), 14 (D14) e 21 (D21) após o tratamento. As análises parasitológicas foram realizadas por meio da contagem de ovos por grama de fezes (OPG), de acordo com a técnica descrita por Gordon e Withlock (1939) modificada por Ueno e Gonçalves (1989).

Foram calculadas as médias aritméticas da contagem de OPG para cada coleta realizada, posteriormente, foi calculado o porcentual de redução de OPG e o porcentual de eficácia dos fármacos (Wood et al., 1995), de acordo com as fórmulas a seguir:

Redução OPG $(\%)=100$ (média de OPG dia zero - média de OPG dia n) / média de OPG dia zero,

Onde; dia n é o dia a ser avaliado $\left(7^{\circ}, 14^{\circ}\right.$, $21^{\circ}$ dia após o tratamento).

Eficácia $(\%)=100$ (média de OPG do grupo controle - média de OPG do grupo tratado) / média de OPG do grupo controle.

Foram realizadas coproculturas a partir de um pool de amostras de cada grupo, seguindo o método descrito por Roberts e O'Sullivan (1950). As larvas infectantes obtidas foram identificadas de acordo com as características morfológicas descritas por Keith (1953). No mesmo dia das coletas de fezes $(0,7,14$ e 21 dias), os animais foram pesados, avaliados quanto ao escore da condição corporal (ECC) (Cezar e Souza, 2007) e o grau de anemia pelo método FAMACHA $^{\oplus}$ (Van Wyk, 2002). Essas avaliações foram realizadas com o intuito de identificar os gêneros presentes no rebanho e suas implicações sobre o desempenho e as características fenotípicas das matrizes, em relação ao nível de infecção e redução ocasionado pelos fármacos.

Todas as variáveis foram submetidas ao teste de Shapiro-Wilker para verificar as características de normalidade. Pelo fato da variável contagem de OPG não ter apresentado tais características, esta foi convertida usando $\log ^{10}(x+1)$. Os dados foram analisados considerando-se $\mathrm{o}$ delineamento inteiramente ao acaso, com quatro tratamentos e 12 repetições. Os dados foram submetidos à análise variância e quando significativos pelo teste $\mathrm{F}$, os efeitos dos grupos químicos foram analisados pelo teste de Tukey, a 5\% de significância.

\section{Resultados e Discussão}

Após análise das coproculturas, foi observada maior frequência do gênero Haemonchus, seguido por Trichostrongylus, Strongyloides e Oesophagostomum. O gênero Haemonchus foi prevalente em todos os grupos e períodos avaliados (dia zero, 7, 14 e 21) independente do anti-helmíntico administrado (Tabela 2). Sendo que, o grupo tratado com ivermectina (97\%) e o controle $(99,25 \%)$ apresentaram as maiores porcentagens desse gênero em questão.

Tabela 2. Larvas infectantes (\%) identificadas nas fezes das matrizes de acordo com o tratamento anti-helmíntico nos dias 0 , 7,14 e 21 .

\begin{tabular}{lccccccccccccccccccccccc}
\hline \multirow{2}{*}{ Gênero } & \multicolumn{1}{c}{ levamisol \% } & \multicolumn{1}{c}{ closantel\% } & \multicolumn{1}{c}{ moxidectina \% } & \multicolumn{1}{c}{ ivermectina \% } & \multicolumn{3}{c}{ Controle \% } \\
\cline { 2 - 3 } & 0 & 7 & 14 & 21 & 0 & 7 & 14 & 21 & 0 & 7 & 14 & 21 & 0 & 7 & 14 & 21 & 0 & 7 & 14 & 21 \\
\hline Haemonchus & 72 & 88 & 95 & 85 & 95 & 89 & 97 & 93 & 90 & 73 & 85 & 82 & 100 & 91 & 100 & 97 & 100 & 100 & 100 & 97 \\
Strongyloides & 20 & 4 & 0 & 8 & 4 & 0 & 0 & 1 & 0 & 24 & 8 & 10 & 0 & 4 & 0 & 1 & 0 & 0 & 0 & 0 \\
Trichostrongylus & 8 & 8 & 5 & 7 & 1 & 11 & 1 & 4 & 10 & 3 & 3 & 5 & 0 & 5 & 0 & 2 & 0 & 0 & 0 & 3 \\
Oesophagostomum & 0 & 0 & 0 & 0 & 0 & 0 & 2 & 2 & 0 & 0 & 4 & 3 & 0 & 0 & 0 & 0 & 0 & 0 & 0 & 0 \\
\hline
\end{tabular}

Os grupos desverminados com closantel e moxidectina apresentaram os quatro gêneros avaliados, sendo que o Oesophagostomum menor proporção, $1 \%$ e $1,75 \%$, respectivamente, em comparação com os outros gêneros. Os grupos que receberam levamisol e moxidectina apresentaram uma percentagem maior de Trichostrongylus e Strongyloides. Demonstrando que há uma ação dos fármacos diferenciada sobre os nematoides.

Quando se observa o gênero de helminto mais frequente de acordo com o princípio ativo e o dia de administração do mesmo, nota-se que houve uma flutuação na porcentagem entre os dias avaliados, sendo que no $14^{\circ}$ dia após aplicação do anti-helmíntico ocorreu um aumento do gênero em ambos os tratamentos experimentais possivelmente pode ser justificado por uma reinfecção dos animais, que se mantiveram sob pastejo contínuo durante o experimento.

Este resultado corrobora com os dados observados por Hammerschmidt et al., (2012) e Melo et al., (2015), onde um novo pico de infecção 
por Haemonchus contortus ocorreu 15 dias após a desverminação de caprinos.

O processo de reinfecção também pode ter influenciado a redução do gênero Haemonchus no $7^{\circ}$ e $21^{\circ}$ dia após a medicação, em virtude do cruzamento entre os nematoides sobreviventes no trato gastrintestinal e os em refugia, sendo que estes nematoides por não terem contato com antihelmínticos, não ativam os genes referentes a resistência anti-helmíntica, diluindo e retardando o processo de seleção de nematoides resistentes nas gerações seguintes (Costa et al., 2011a).

O grupo controle no $21^{\circ}$ dia apresentou $3 \%$ do gênero Trichostrongylus, esse gênero tende a compensar a baixa produção de ovos com a manutenção de grandes populações de adultos, estando presente no hospedeiro sem ser notado, ao contrário do gênero Haemonchus que possui alta taxa reprodutiva, sendo mais expressos e quantificados nas avaliações (Melo et al., 2015).

Resultados semelhantes foram encontrados por Costa et al. (2011b) na região oeste do Rio
Grande do Norte, quando avaliaram os efeitos do tratamento com closantel e ivermectina sobre a carga parasitária de ovinos. Incidência semelhante também observada por Costa et al. (2017) quando avaliaram a eficácia de diferentes princípios ativos em raças de ovinos lanados, na região sul do Brasil.

Houve diferença significativa $(\mathrm{P}<0,05)$ na OPG dos animais no $7^{\circ}, 14^{\circ}, 21^{\circ}$ dias após a administração dos diferentes princípios ativos, e as maiores médias foram observadas nos animais tratados com ivermectina (Tabela 3), demonstrando que os nematoides gastrintestinais já possuem resistência anti-helmíntica para esse grupo químico. No dia zero todos os grupos apresentaram alta contagem de OPG, resultado considerado normal, pois os mesmos não tinham sido tratados por um período de dois meses, e além disso, o experimento ocorreu em período chuvoso, o que causa aumento na umidade que proporciona um microclima ideal para o desenvolvimento de lavas infectantes de parasitos no pasto.

Tabela 3. Médias e desvio padrão da contagem de OPG e redução de OPG (\%) de acordo com o tratamento antihelmíntico nos dias $0,7,14$ e 21 .

\begin{tabular}{cccccccc}
\hline $\begin{array}{c}\text { Princípio } \\
\text { Ativo }\end{array}$ & $\begin{array}{c}\text { OPG } \\
\text { (Dia 0) }\end{array}$ & $\begin{array}{c}\text { OPG } \\
\text { (Dia 7) }\end{array}$ & $\begin{array}{c}\text { ROPG } \\
\text { \% }\end{array}$ & $\begin{array}{c}\text { OPG } \\
\text { (Dia 14) }\end{array}$ & $\begin{array}{c}\text { ROPG } \\
\text { \% }^{\text {Dia }}\end{array}$ & $\begin{array}{c}\text { OPG } \\
\text { (Dia 21) }\end{array}$ & $\begin{array}{c}\text { ROPG } \\
\text { \% }\end{array}$ \\
\hline Levamisol & $4391,7 \pm 4388^{\mathrm{ab}}$ & $175 \pm 158^{\mathrm{bc}}$ & 96,0 & $441,7 \pm 422^{\mathrm{b}}$ & 90,0 & $358,3 \pm 221^{\mathrm{b}}$ & 91,8 \\
Closantel & $2433,3 \pm 3223^{\mathrm{b}}$ & $350 \pm 400^{\mathrm{bc}}$ & 85,6 & $166,7 \pm 262^{\mathrm{b}}$ & 93,1 & $366,7 \pm 433^{\mathrm{b}}$ & 84,9 \\
Moxidectina & $5833,3 \pm 5138^{\mathrm{a}}$ & $41,6 \pm 55,5^{\mathrm{b}}$ & 99,3 & $141,0 \pm 455^{\mathrm{b}}$ & 97,6 & $225,0 \pm 200^{\mathrm{b}}$ & 96,1 \\
Ivermectina & $7858,3 \pm 6880^{\mathrm{a}}$ & $1766 \pm 1422^{\mathrm{a}}$ & 77,5 & $1100 \pm 883^{\mathrm{ab}}$ & 86,0 & $2208,0 \pm 2128^{\mathrm{a}}$ & 71,9 \\
Controle & $2690 \pm 1426^{\mathrm{ab}}$ & $4000 \pm 3540^{\mathrm{a}}$ & $-48,7$ & $3980 \pm 2932^{\mathrm{a}}$ & $-47,9$ & $3380,0 \pm 3188^{\mathrm{a}}$ & $-25,6$ \\
\hline
\end{tabular}

Médias seguidas de letras distintas na coluna diferem pelo teste de Tukey $(\mathrm{P}<0,05)$. Redução de OPG (ROPG \%).

Após a aplicação dos diferentes antihelmínticos houve redução da OPG dos animais tratados em todos os períodos menos para o grupo controle, o que já era esperado devido esse grupo não ter recebido nenhum tratamento. Semelhante aos resultados da presente pesquisa, Costa et al. (2017), também encontraram redução na OPG de animais tratados com os grupos químicos levamisol, closantel e moxidectina, os mesmos autores não observaram redução para o grupo controle.

A ivermectina foi ineficaz na redução da OPG, com valores inferiores a $90 \%$ em todos os dias avaliados (Tabela 4). A eficácia abaixo de 90\% é suficiente para pressupor a resistência dos helmintos a essa droga (Chagas e Veríssimo, 2008; Santos et al., 2017).

Tabela 4. Eficácia (\%) dos princípios ativos utilizados no controle de parasitos gastrintestinais de ovinos nos dias 7,14 e 21 pós-tratamento.

\begin{tabular}{lccc}
\hline \multicolumn{1}{c}{ Princípio Ativo } & Dia 07 & Dia 14 & Dia 21 \\
\hline Levamisol & 95,6 & 89,0 & 89,4 \\
Closantel & 91,2 & 95,8 & 89,1 \\
Moxidectina & 99,0 & 96,4 & 93,3 \\
Ivermectina & 55,8 & 72,4 & 34,7 \\
\hline
\end{tabular}

A moxidectina apresentou eficácia de $99 \%$, $96,4 \%$ e $93,3 \%$, respectivamente, nos dias 7, 14 e 21 pós-tratamento. Anti-helmíntico com porcentagem de redução acima de $98 \%$ é considerado eficaz, segundo a portaria $\mathrm{N}^{\circ} 48$, do Ministério da Agricultura, Pecuária e 
Abastecimento (1997). Os anti-helmínticos a base de avermectinas e milbemicinas que fazem parte do grupo das lactonas macrocíclicas tem sido utilizadas em larga escala no Brasil, acarretando em vários relatos de resistência a essa droga (Rosalinsk-Moraes et al., 2007; Costa et al., 2017), porém, vem sendo pouco utilizada na região Nordeste do Brasil, o que pode ser uma opção de tratamento para as helmintoses.

De acordo com essa mesma portaria do MAPA (1997) para substâncias químicas dotadas de atividade antiparasitária, os fármacos que apresentam eficácia entre $90 \%$ e $98 \%$, são considerados efetivos, sendo o levamisol e o closantel agrupados nessa categoria. Porém, apesar de serem considerados efetivos, ainda mantém em torno de $10 \%$ da população parasitária no hospedeiro, selecionando assim os indivíduos resistentes.

Não foi observada diferença no peso final das matrizes ovinas tratadas com os diferentes grupos químicos ( $\mathrm{P}>0,05)$, com média de $39,5 \mathrm{~kg}$, isso ocorreu devido ao curto período de avaliação que foi de apenas de 21 dias. O parasitismo tem efeitos severos na produção, com destaque para o Haemonchus spp., sendo responsáveis por grandes perdas na produção de pequenos ruminantes em todo o mundo (Soli et al., 2010), podendo reduzir de 20 a $60 \%$ o ganho de peso.

Os valores de ECC nas matrizes tratadas com ivermectina, levamisol e o grupo controle, teve maior concentração nos graus 2,5 e 3 . Já nas matrizes tratadas com moxidectina, apresentaram ECC entre 2,5 e 3,5. Nas matrizes que receberam o closantel foram agrupadas entre 2,5 a 4,5 com uma maior dispersão dos dados (Figura 1). Os animais tratados com closantel e moxidectina foram os que apresentaram melhores ECC, mantendo o peso e a composição muscular e adiposa.
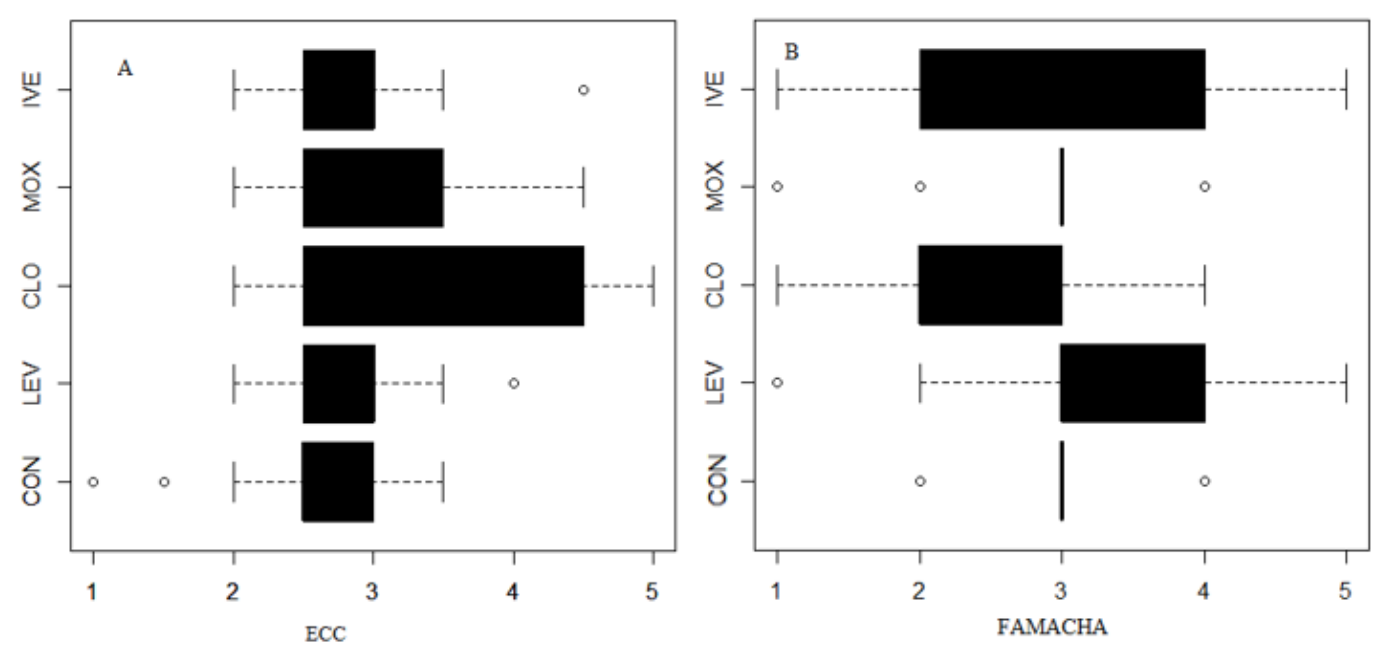

Figura 1. Distribuição do escore de condição corporal (A) e grau de FAMACHA (B) de matrizes ovinas tratadas com diferentes princípios ativos. Ivermectina (IVE), moxidectina (MOX), closantel (CLO), levamisol (LEV), e grupo controle (CON) não tratado.

É importante que o ECC quando utilizado com a finalidade de indicação indireta de infecções parasitárias, seja associada a outras variáveis fenotípicas, tais como, grau de anemia e/ou exame laboratorial, para uma melhor acurácia, visto que esta avaliação é uma medida subjetiva, e além disso, em curtos períodos de avaliação o parasitismo pode não ter influência sobre essa variável, sendo esta uma ferramenta a ser utilizada com objetivo de controle e prevenção por longos períodos na propriedade.

$\mathrm{Na}$ fase de pós aleitamento, as matrizes tendem a recuperar o sistema reprodutivo e suas reservas energéticas para a futura prenhez, e o ECC é uma das variáveis que melhor representa a condição do animal. Roberto et al. (2018) observaram que, conforme aumenta o ECC, aumenta a percentagem de ovelhas prenhes, matrizes com ECC 3,0 e 4,0 apresentaram 92\% e 98\% de prenhez, respectivamente. Portanto, o uso de anti-helmínticos ineficazes em matrizes pode contribuir para elevadas cargas parasitárias, comprometendo o desempenho produtivo $\mathrm{e}$ reprodutivo do rebanho.

Na avaliação do grau de anemia pelo método FAMACHA foi observado uma maior concentração dos animais entre 2 e 4 (Figura 1). Sendo que os animais do grupo tratado com 
ivermectina os que apresentaram uma maior dispersão nesse intervalo. Isso ocorreu por esse anti-helmíntico ter sido o menos eficaz, associado a infecção parasitária mista e a alta percentagem do gênero Haemonchus, que possui ação hematófaga e espoliativa.

Os animais tratados com closantel apresentaram-se concentrados entre os graus 2 e 3 , que corresponde a animais não anêmicos, mesmo apresentando infecções mistas, a carga parasitária em algumas avaliações foi baixa. Os animais do grupo tratado com moxidectina e o grupo controle apresentaram grau 3. O grupo controle apesar da infecção maciça que apresentavam e praticamente composta por $100 \%$ de Haemonchus contortus, pode ter reagido melhor por estar na fase final do balanço energético negativo, fase fisiológica que proporciona as matrizes imunidade baixa. $\mathrm{O}$ levamisol possui um efeito modulador no sistema imune tendo alta eficiência no controle de Haemonchus contortus (Costa et al., 2017). Apesar do closantel ter apresentado baixa eficácia (Tabela 3) poucos animais apresentaram FAMACHA 3.

$\mathrm{O}$ grau de anemia pelo FAMACHA $^{\odot}$ tem correlação positiva com a carga de endoparasitos hematófagos, sendo uma avaliação de fácil aplicação nos rebanhos. A eficácia do método é maior quando a frequência de Haemonchus ssp. representa pelo menos $60 \%$ dos gêneros de helmintos, pois esse é o principal parasito que causa anemia nos pequenos ruminantes (Chagas et al., 2007; Bath e Van Wyk, 2009). Sendo assim, se torna importante o monitoramento dos gêneros por coproculturas a cada 60 dias, a fim de verificar se a prevalência do Haemonchus ssp. no rebanho (Silva et al., 2017).

\section{Conclusão}

O princípio moxidectina mostrou-se eficaz na redução de nematoides gastrintestinais em matrizes ovinas, podendo ser indicado no controle desses parasitos. O Haemonchus spp. se mostrou resistente aos fármacos utilizados, e prevalente no rebanho.

\section{Conflito de Interesse}

Os autores declaram não existir conflito de interesse.

\section{Comitê de ética}

O trabalho foi aprovado pela Comissão de Ética no Uso de Animais - CEUA da UFRN, sob protocolo $n^{\circ}$ 055/2016.

\section{Referências}

Ahid, S.M.; Suassuna, A.C.D.; Maia, M.B.; Costa, V.M.M.; Soares, H.S. Parasitos gastrintestinais em caprinos e ovinos da região oeste do Rio Grande do Norte, Brasil. Ciência Animal Brasileira, 9(1): 212-218, 2008.

Amarante, A.F.T. Nematoides gastrintestinais em ovinos In: Doenças parasitárias de caprinos e ovinos: epidemiologia e controle. Brasília: Embrapa, 2009. p. 19-6.

Bath, G.F.; Van Wyk, J.A.; The five point check for targeted selective treatment of internal parasites. Small Ruminant, 86(1), 6-13, 2009.

BRASIL. Ministério da Agricultura, Pecuária e Abastecimento. Instrução Normativa $\mathbf{N}^{\mathbf{0}} \mathbf{4 8}$, de 1997. Disponível em: <http://sistemasweb.agricultura.gov.br/sislegi s/action/detalhaato.do?method=visualizarato portalmapa\&chave $=72818869>$. Acesso em: 15 dez. 2017.

Cezar, M.F.; Sousa, W.H.; Carcaças ovinas e caprina: obtenção: avaliação, classificação. João Pessoa: Agropecuária tropical, 2007. 232 p.

Chagas, A.C.S.; Carvalho, C.O.; Molento, M.B. Método FAMACHA: um recurso para o controle da verminose em ovinos. Circular Técnica 52. São Carlos: Embrapa Pecuária Sudeste, 2007.

Chagas, A.C.S.; Veríssimo, C.J. Principais enfermidades e manejo sanitário de ovinos. Embrapa Pecuária Sudeste. 2008, 1: 70p.

Costa, K.M.F.M.; Ahid, S.M.M.; Viera, L.S.; Vale A.M.; Soto-Blanco, B. Efeitos do tratamento com closantel e ivermectina na carga parasitária, no peril hematológico e bioquímico sérico e no grau Famacha de ovinos infectados com nematódeos. Pesquisa Veterinária Brasileira, 31(12): 1075-1082, 2011a.

Costa, P.T.; Costa, R.T.; Mendonça, G.; Vaz, R.Z. Eficácia anti-helmíntica comparativa do nitroxinil, levamisol, closantel, moxidectina e fenbendazole no controle parasitário em ovinos. Boletim de Indústria Animal. 74(1): 72-78, 2017.

Costa, V.M.M.; Simões, S.V.D.; Riet-Correa, F. Controle das parasitoses gastrintestinais em ovinos e caprinos na região semiárida do Nordeste do Brasil. Pesquisa Veterinária Brasileira, 31(1): 65-71, 2011b. 
Duarte, E.R.; Silva, R.B.; Vasconcelos, V.O.; Nogueira, F.A.; Oliveira, N.J.F. Diagnóstico do controle e perfil de sensibilidade de nematoides de ovinos ao albendazol e ao levamisol no norte de Minas Gerais. Pesquisa Veterinária Brasileira, 32(2): 147-152, 2012.

Gordon, H.M.C.L.; Whitlock, A.V. A new technique for counting nematode eggs in sheep feces. Journal Council Scientific Industry Research Australia, 12: 50-52, 1939.

Hammerschmidt, J.; Bier, D.; Fortes, F.S.; Warzensaky, P.; Bainy, A.M.; Macedo, A.A.S.; Molento, M.B. Avaliação do sistema integrado de controle parasitário em uma criação semi-intensiva de caprinos na região de Santa Catarina. Arquivos Brasileiros de Medicina Veterinária e Zootecnia, 64(4): 927-934, 2012

IBGE - Instituto Brasileiro de Geografia e Estatística. Banco de dados agregados. 2017. Rio de Janeiro. Disponível em: $<$ https://brasilemsintese.ibge.gov.br/agropecu aria/efetivos-da-pecuaria.html/>. Acesso em: 02 dez. 2017.

INMET - Instituto Nacional de Metereologia. Banco de dados. 2017. Brasília. Disponível em: <http://www.inmet.gov.br/portal/>. Acesso em: 15 sez. 2017.

Keith, R.K. The differentiation of infective larvae of some common nematode parasites of cattle. Australian Journal Zoology, 1: 223-235, 1953.

Melo, V.F.P.; Pinheiro, R.S.B.; Homem Junior, A.C.; Américo, J.H.P.; Santos, V.C.; Rosestolato, L.L.R. Manejo de antihelmínticos no controle de infecções gastrintestinais em cabras. Revista Brasileira de Saúde e Produção Animal, 16(4): 916924, 2015.

Molento, M.B. Resistência de helmintos em ovinos e caprinos. Revista Brasileira de Parasitologia, 13: 82-86, 2004.

NRC - National Research Council. Nutrients requirements of sheep. Washington: National Academies Press, 2007.

Pinheiro, R.R.; Gouveia, A.M.G.; Alves, F.S.F.; Haddad, J.P.A. Aspectos epidemiológicos na caprinocultura cearense. Arquivo Brasileiro de Medicina Veterinária e Zootecnia, 52(5): 534-543, 2000.
Roberto, F.F.S.; Difante, G.S.; Zaros, L.G.; Gurgel, A.L.C. Nematoides gastrintestinais na ovinocultura de corte sob regime de pastejo. PUBVET, 12(4): 1-12, 2018.

Roberts, F.H.S.; O'sullivan P.J. Methods for egg counts and larval cultures for Strongyles infesting the gastro-intestinal tract of cattle. Crop and Pasture Science, 1: 99-102, 1950.

Rosalinsk-Moraes, F.; Morreto, L.H.; Bresolin, W.S.; Gabrielli, I.; Kafer, L.; Zanchet, I.K. Resistência anti-helmíntica em rebanhos ovinos da região da associação dos municípios do Alto Irani (AMAI) oeste de Santa Catarina. Ciência Animal Brasileira, 8(1): 559-565, 2007.

Santos, J.M.L.; Vasconcelos, J.F.; Frota, G.A.; Ribeiro, W.L.C.; André, W.P.P.; Vieira, L.S Marcel Teixeira, M.; Bevilaqua, C.M.L.; Jomar Patrício Monteiro, J.P. Haemonchus contortus $\beta$-tubulin isotype 1 gene $\mathrm{F} 200 \mathrm{Y}$ and F167Y SNPs are both selected by ivermectin and oxfendazole treatments with differing impacts on anthelmintic resistance. Veterinary Parasitology, 248(1): 90-95, 2017.

Silva, D.G.; Pilatti, J.A.; Menezes, B.M.; Brum, L.P.; Goulart Netto, C.; Martins, A.A. Eficácia anti-helmíntica comparativa entre diferentes princípios ativos em ovinos jovens. PUBVET, 11(4): 356-362, 2017.

Silva, D.G.; Menezes, B.M.; Bettencourt, A.F.; Frantz, A.F., Corrêa, M.R.; Ruszkowski, G.; Martins, A.A.; Brum, L.P.; Hirschmann, L.C. Método FAMACHA ${ }^{\circledR}$ como ferramenta para verificar a infestação parasitária ocasionada por Haemonchus spp. em ovinos. PUBVET, 11(10): 1015-1021, 2017.

Soli, F.; Terrill, T.H.; Shaik, S.A.; Getz, W.R.; Miller, J.E.; Vanguru, M. Eficacy of copper oxide wire particles against gastrointestinal nematodes in sheep and goats. Veterinary Parasitology, 168(1): 93-96, 2010.

Thorthwaite, C.W. An approach toward a rational classification of climate. Geographic Review, 38: 55-93, 1948.

Ueno, H.; Gonçalves P.C. Manual para diagnóstico das helmintoses de ruminantes. Japan Internacional Cooperation Agency (JICA), 4: 1-143, 1998.

Van Wyk, J.A.; Bath, G.F. The FAMACHA system for managing haemonchosis in sheep and goats by clinically identifying individual 
animals for treatment. Veterinary Research, 33(5): 509-529, 2002.

Wood, I.B.; Amaral, N.K.; Bairden, K.; Duncan, J.L.; Kassai, T.; Malone, J.B. World Association for the Advancement of
Veterinary Parasitology second edition of guidelines for evaluating the efficacy of anthelmintics in ruminants (bovine, ovine, caprine). Veterinary Parasitology, 58: 181213, 1995. 